\title{
Hepatitis B and HIV co-infection in pregnant women: Indication for routine antenatal hepatitis B virus screening in a high HIV prevalence setting
}

\author{
N V Thumbiran, ${ }^{1} \mathrm{MB}$ ChB, Dip HIV/AIDS Clin Man; D Moodley, ${ }^{2} \mathrm{BSc}, \mathrm{MMedSc}, \mathrm{PhD}$; \\ R Parboosing, ${ }^{1}$ MB ChB, FCPath (Virology), MMed, MS (Epi); P Moodley, ${ }^{1}$ MB ChB, FCPath (Virology) \\ ${ }^{1}$ Department of Virology, National Health Laboratory Service, University of KwaZulu-Natal, Durban, South Africa \\ ${ }^{2}$ Women's Health and HIV Research Unit, Department of Obstetrics and Gynaecology, University of KwaZulu-Natal, Durban, South Africa
}

Corresponding author: N V Thumbiran (nthumbiran@gmail.com)

\begin{abstract}
Background. Sub-Saharan Africa is endemic for hepatitis B virus (HBV) and human immunodeficiency virus (HIV) infections. HBV/HIV co-infection in women of reproductive age is of clinical and public health importance because these women constitute a significant reservoir for horizontal and perinatal HBV transmission. Childhood HBV vaccination from 6 weeks of age protects most children against chronic HBV infection. However, infants born to HBV/HIV co-infected women are more likely to be infected perinatally, with an increased risk of chronic hepatitis, than infants born to HBV mono-infected women.

Objectives. The aim of our study was to establish the prevalence of HBV infection and HBV/HIV co-infection in pregnant women in KwaZulu-Natal, South Africa, to inform antenatal HBV screening and childhood immunisation policies in South Africa.

Methods. Stored plasma specimens obtained from 570 pregnant women were tested for hepatitis B surface antigen (HBsAg) and HBV infectivity, as characterised by the presence of hepatitis B e antigen (HBeAg) and/or HBV DNA load.

Results. The antenatal HIV prevalence and HBsAg prevalence in this study were $41.6 \%$ and $5.3 \%$ (95\% confidence interval (CI) $3.4-7.1$ ), respectively. Overall, 3.1\% (95\% CI 1.7 - 4.6) of pregnant women were HBV/HIV co-infected, with HBeAg positivity and the HBV DNA load being significantly higher in co-infected women.

Conclusion. We report a 5.3\% HBV prevalence and a 3.1\% HBV/HIV co-infection prevalence in pregnant women from this HIV-endemic region. Routine antenatal HBV screening will allow early identification of neonates who require HBV active-passive immunoprophylaxis at birth. This strategy, together with antenatal antiretrovirals, will reduce the risk of perinatal HBV transmission, especially in high-risk HBV/ HIV co-infected pregnant women.
\end{abstract}

S Afr Med J 2014;104(4):307-309. DOI:10.7196/SAMJ.7299

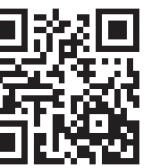

An estimated 240 million chronic hepatitis B virus (HBV) infections have been reported worldwide despite the availability of an effective vaccine. ${ }^{[1]}$ South Africa (SA) introduced the HBV vaccine into the national Expanded Programme on Immunisation (EPI) in 1995. ${ }^{[2]}$ The vaccine is given to infants at 6,10 and 14 weeks of age. ${ }^{[2]}$ Adults are not routinely immunised against HBV, so women born before 1995 would have no vaccine immunity against HBV. Between 1995 and 1999, 31\% of mothers of 8 - 72-month-old children in an SA study had evidence of current or past exposure to HBV. ${ }^{[2]}$

Co-infection with HBV and the human immunodeficiency virus (HIV) in sexually active women of reproductive age is of significant public health importance because such women are at risk of ongoing HBV transmission, both horizontally and perinatally. ${ }^{[3]}$ A fixeddose combination (FDC) antiretroviral (ARV) regimen consisting of tenofovir (TDF), emtricitabine (FTC) and efavirenz (EFV) is currently recommended for all HIV-positive pregnant women regardless of their $\mathrm{CD}^{+}$cell count. ${ }^{[4]}$ Since TDF and FTC are also effective in the treatment of $\mathrm{HBV}$ infection, these prevention of mother-to-child transmission (PMTCT) guidelines are the first in SA to offer simultaneous prevention of HIV and HBV perinatal transmission. ${ }^{[5]}$ In women who do not meet the eligibility criteria for ongoing treatment, i.e. a $\mathrm{CD}^{+}$count of $\leq 350$ cells/ $\mu \mathrm{l}$, the FDC regimen is intended to be prophylactic and discontinued 1 week after cessation of breastfeeding. ${ }^{[4]}$ The guidelines therefore recommend $\mathrm{HBV}$ screening prior to discontinuing the ARV regimen to avoid maternal HBV reactivation. ${ }^{[4]}$ The potential disadvantage of this delayed screening is failure to identify the HBV-exposed neonate and protect it at birth against developing chronic HBV infection.

Childhood immunisation programmes, which include $\mathrm{HBV}$ vaccination from 6 weeks of age, protect the majority of children and young adults against chronic hepatitis and liver-related mortality. Infants born to HBV/HIV co-infected women are, however, more likely to be infected perinatally and are at greater risk of developing chronic hepatitis than infants born to HBV mono-infected women. ${ }^{[3]}$ Routine antenatal HBV screening is not offered in SA, yet local studies reported $\mathrm{HBV}$ prevalence rates ranging between $3.2 \%$ and $7.4 \%$ in HIV-infected pregnant women. ${ }^{[3,6]}$ The aim of our study was to establish the prevalence of HBV infection and HBV/HIV co-infection in pregnant women in KwaZulu-Natal $(\mathrm{KZN})$, to inform antenatal HBV screening and childhood immunisation policies in SA.

\section{Methods}

Stored $\left(-70^{\circ} \mathrm{C}\right)$ plasma specimens were tested for $\mathrm{HBV}$ markers in a retrospective analysis of pregnant women who participated in an HIV sero-incidence study in KZN between March and December 2009. ${ }^{[7]}$ Consent for 'storage of plasma intended for use in further research' was obtained from participants at enrolment into the original study. The process of participant recruitment, eligibility 
criteria and HIV testing procedures have been described previously. ${ }^{[7]}$ Plasma aliquots from 570 stored specimens were selected based on an estimated HBV prevalence of 5.5\%. ${ }^{[8]}$ The participants' ages, HIV status and $\mathrm{CD} 4^{+}$counts were extracted from the original study database.

\section{Laboratory analysis}

Frozen plasma specimens were thawed and tested for hepatitis B surface antigen ( $\mathrm{HBs} A g$ ) using an enzyme-linked immunosorbent assay (ELISA) from Siemens Healthcare Diagnostics (Tarrytown, NY, USA). If HBsAg-positive, specimens were further tested for hepatitis B e antigen (HBeAg), also using an ELISA from Siemens Healthcare Diagnostics, and for quantitative HBV DNA using the COBAS AmpliPrep/COBAS TaqMan HBV Test, version 2.0, from Roche Molecular Systems Inc. (Branchburg, NJ, USA). HBV infection was characterised by a positive HBsAg result and hepatitis B viral infectivity by the presence of HBeAg and/or HBV DNA quantification.

Specimens with low HBsAg and $\mathrm{HBeAg}$ index values were not retested for confirmation owing to insufficient specimen volumes. HBsAg specimens with index values of $\geq 1$ and $\leq 50$ were further neutralised to confirm the presence of HBsAg, and specimens that produced technically invalid results were excluded from the analysis. HBeAg index values of $\geq 10$ were interpreted as positive.

The linear range for HBV DNA quantification was $20-1.7 \times 10^{8} \mathrm{IU} / \mathrm{ml}$. Specimens with an insufficient volume were diluted and the final results were calculated by multiplication using the dilution factor.

\section{Statistical analysis}

Prevalence rates for HBV infection and HBV/HIV co-infection were reported as point estimates with $95 \%$ confidence intervals (CIs). Differences in proportions were determined by the chi-square test (and Fisher's exact test, where Cochrane's criterion was not fulfilled). Two-tailed non-parametric procedures were used to compare HBV DNA load between groups. A $p$-value of $<0.05$ was regarded as significant. SAS 9.3 (SAS Institute Inc., Cary, NC, USA) was used for statistical analysis.

\section{Ethical considerations}

Ethical approval was obtained from the University of KwaZulu-Natal Biomedical Research Ethics Committee (BE028/12).

\section{Results}

Five hundred and seventeen of 570 (90.7\%) pregnant women (median age 23 years, range 16 - 47 years) had their HIV status documented; 215 (41.6\%) were HIV-positive.

Thirty tested positive for HBsAg, resulting in a point prevalence estimate of 5.3\% (95\% CI 3.4 - 7.1). Of these women, 6 were HBeAg-positive (20.0\%; 95\% CI 5.7 - 34.3). Twenty-five HBsAg-positive specimens (83.3\%) had detectable HBV DNA, ranging from 20 to $>1.7 \times 10^{8} \mathrm{IU} / \mathrm{ml}$ with a median $\log _{10}$ viral load of 3.0 (interquartile range (IQR) 2.3).

Although not statistically significant, more HIV-infected women than HIV-uninfected women tested HBsAg-positive $(p=0.2048)$ (Table 1). Overall, 16 of 509 (3.1\%; 95\% CI 1.7 - 4.6) pregnant women with HIV and HBsAg results were HBV/HIV co-infected. $\mathrm{HBeAg}$ positivity was significantly more common in HIV-infected than in HIVuninfected women $(p=0.0185)$ (Table 1). All $16 \mathrm{HBV} / \mathrm{HIV}$ co-infected women had a detectable HBV DNA load ranging from 45 to $>1.7 \times 10^{8} \mathrm{IU} / \mathrm{ml}$ with a median $\log _{10}$ viral load of 3.3 (IQR 3.9), which was significantly higher than in HIV-uninfected women (Table 1).

A CD4 $4^{+}$cell count was available for $76 / 215$ (35.4\%) HIV-positive pregnant women, with a median of 328 cells/ $\mu$ l (range 45 - 822 ; IQR 183). Of these women, $41(53.9 \%)$ had a $\mathrm{CD} 4{ }^{+}$count of $\leq 350$ cells $/ \mu$ and would have been eligible for lifelong ARV treatment. Four of the 41 women $(9.7 \%)$ with a $\mathrm{CD} 4^{+}$ count of $\leq 350$ cells $/ \mu$ l were HBV-co-infected, while none of the women with a $\mathrm{CD} 4^{+}$count of $>350$ cells $/ \mu \mathrm{l}$ was co-infected $(p=0.119$ using Fisher's exact test, two-tailed).

\section{Discussion}

We report an estimated HBV prevalence of $5.3 \%$ in pregnant women attending primary health clinics in KZN. It is highly likely that most of the pregnant women in our study had missed the childhood EPI vaccination. ${ }^{[2]}$ One in 5 (20\%) of these HBVinfected women had active viral replication, as indicated by the presence of HBeAg. We further report a $7.4 \%$ HBsAg prevalence in HIV-infected pregnant women in KZN, which is higher than the $4.3 \%, 5.9 \%$ and $6.2 \%$ reported from studies in HIVinfected pregnant women in North West, the Western Cape and Limpopo provinces, respectively. ${ }^{[3,6]}$ This regional variation in HBV prevalence may be due to various cultural and environmental factors that modify HBV transmission in diverse ethnic groups. ${ }^{[9]}$ The non-significant difference in HBV prevalence between HIV-infected and uninfected pregnant women demonstrated in our study is similar to that reported in other SA studies and may be explained by early childhood cryptic transmission of HBV rather than shared routes of HIV and HBV transmission in adulthood. ${ }^{[6]}$

The 3.1\% HBV/HIV co-infection rate in our study population is clinically significant in a setting with a high HIV burden. HBV/ HIV co-infected pregnant women require complex management, since the impact on the mother and the infant must be considered. The FDC ARV regimen (TDF/ FTC/EFV) currently recommended for all HIV-positive pregnant women benefits the mother with HBV co-infection and also offers protection against HBV infection to the exposed neonate by reducing the maternal HBV DNA load, which is an independent risk factor for perinatal transmission. ${ }^{[5,10,11]}$ This strategy provides a great advantage over the past zidovudine and single-dose nevirapine (NVP) PMTCT regimen in which a single postpartum dose of TDF and FTC was given. ${ }^{[12]}$ While this was intended to protect the mother from developing NVP resistance, it offered no benefit to the mother or her exposed neonate if she was also HBV-infected. The use of antenatal ARVs against hepatitis B has been shown to be relatively safe and may compensate for immunoprophylaxis failure, especially in resource-limited settings where early access to immunisation is not always available..$^{[5,10,13]}$ The new national PMTCT guidelines recommend maternal HBV screening of all HIV-infected women

Table 1. Distribution of hepatitis B virus laboratory markers in HIV-infected and -uninfected pregnant women

\begin{tabular}{llll}
\hline Laboratory markers & HIV-infected & HIV-uninfected & p-value \\
\hline HBsAg+, $n(\%)$ & $16 / 215(7.4)$ & $14 / 294^{*}(4.8)$ & $0.2048^{\dagger}$ \\
HBeAg+, $n(\%)$ & $6 / 16(37.5)$ & $0 / 14(0.0)$ & $0.0185^{\ddagger}$ \\
HBV DNA load, median (IQR) & $3.3 \log _{10}(3.9)$ & $1.5 \log _{10}(2.1)$ & $0.0058^{\varsigma}$ \\
& & \\
HBsAg = hepatitis B surface antigen; HBeAg = hepatitis B e antigen; HBV = hepatitis B virus; + = positive; \\
IQR = interquartile range. \\
'Excludes 8 HBsAg invalid results. \\
'Chi-squared test. \\
'Fisher's exact test. \\
'Two-tailed non-parametric test.
\end{tabular}


receiving FDC prior to discontinuing the prophylactic ARV regimen at cessation of breastfeeding. ${ }^{[4]}$ This strategy protects HBV/HIV co-infected women from developing hepatitis flares. However, postnatal HBV screening, as opposed to antenatal screening, would unfortunately be too late to identify HBV-exposed neonates who would have required $\mathrm{HBV}$ immunisation at birth. ${ }^{[4,14]}$

Furthermore, it is highly likely that the $5.3 \%$ of neonates in our study who were HBV-exposed were not vaccinated at birth as recommended, since $\mathrm{HBV}$ screening in pregnancy is not routinely available and the HBV infection in these women would have gone undetected. The higher HBV infectivity demonstrated in HBV/ HIV co-infected pregnant women in our study is consistent with other recent studies, and suggests that mother-to-child transmission (MTCT) of HBV may be more common in this country than has previously been thought. ${ }^{[3,6,15]}$ Perinatal HBV transmission often results in an asymptomatic neonatal infection, and $70-90 \%$ of these neonates remain chronically infected if HBV immunoprophylaxis is not given at birth. ${ }^{[16]}$ They will subsequently contribute to the reservoir of asymptomatic HBV carriers as adults. ${ }^{[16]}$ Inclusion of the HBV vaccine into the South African EPI has reduced the childhood HBV prevalence, but vaccination initiated at 6 weeks of age is inadequate to protect against perinatal HBV transmission. ${ }^{[3,6]}$ Immunoprophylaxis failure has been reported despite the suppression of HBV DNA load with antenatal ARVs, suggesting that other maternal and obstetric factors may influence transmission. ${ }^{[11]}$ Inability to prevent perinatal transmission from high-risk HBV/HIV co-infected mothers will result in a residual pool of $\mathrm{HBV}$-infected children who will be a source of ongoing transmission to others, and this may impede current vaccination efforts.

Our failure to investigate occult HBV infections may have meant that we underestimated HBV prevalence. The rate of occult HBV infection in HIV-infected individuals is estimated to be $33 \% .{ }^{[17]}$ Testing for occult HBV infection could not be performed in our retrospective analysis owing to inadequate specimen volume for hepatitis B core antibody testing and confirmatory molecular testing. However, the unknown clinical significance of occult HBV infection, particularly its role in HBV transmission, together with the high cost of HBV molecular screening for an accurate diagnosis, means that it is unlikely to be included in a public health policy. ${ }^{[18]}$

Routine antenatal HBV screening in pregnant women in SA will allow for the timeous initiation of infant immunoprophylaxis and identification of HBV-exposed neonates who require postimmunisation serological testing to confirm HBV status. Active and passive immunisation given to the neonate within $12-24$ hours of birth (before the development of HBV surface antigenaemia) was shown to prevent more than $95 \%$ of perinatal transmissions. ${ }^{[5,14]}$ However, in a developing country where many women may not access antenatal care, initiating the HBV vaccination schedule in all infants at birth rather than at 6 weeks could aid in preventing perinatal HBV transmission.

\section{Conclusion}

We report an estimated 5.3\% prevalence of HBV infection and a $3.1 \%$ prevalence of $\mathrm{HBV} / \mathrm{HIV}$ co-infection in pregnant women in this HIV-endemic region. It is highly likely that most women of reproductive age who are also HIV-infected have not received childhood HBV immunisation. While the current HIV PMTCT guidelines provide potent antenatal ARVs with the potential to suppress HBV DNA load and decrease vertical HBV transmission in high-risk HBV/HIV co-infected women, approximately $4.8 \%$ of neonates born to HIV-uninfected women are at risk of perinatal HBV infection. Routine antenatal serological screening for HBV will identify all HBV-exposed neonates and allow active-passive HBV immunoprophylaxis to be initiated at birth, thereby reducing the risk of vaccine failure. There are no formal guidelines for PMTCT of HBV, but antenatal antiviral therapy in combination with immunoprophylaxis initiated at birth will decrease the risk of MTCT of HBV, as shown in many studies. This strategy may, in turn, reduce infant morbidity and mortality related to $\mathrm{HBV}$ infection as well as decrease the reservoir for ongoing horizontal HBV transmission. Our study confirms other SA reports and highlights the need for SA childhood immunisation policies to be revisited. Prospective studies assessing the impact of the current PMTCT guidelines on MTCT of $\mathrm{HBV}$ are needed in the future.

Acknowledgement. Funding was received from the National Health Laboratory Service Research Trust.

\section{References}

1. Ott JJ, Stevens GA, Groeger J, Wiersma ST. Global epidemiology of hepatitis B virus infection: New estimates of age-specific HBsAg seroprevalence and endemicity. Vaccine 2012;30(12):2212-2219. http://dx.doi.org/10.1016/j.vaccine.2011.12.116

2. Tsebe KV, Burnett RJ, Hlungwani NP, Sibara MM, Venter PA, Mphahlele MJ. The first five years of universal hepatitis B vaccination in South Africa: Evidence for elimination of HBsAg carriage in under 5-year-olds. Vaccine 2001;19(28-29):3919-3926. [http://dx.doi.org/10.1016/S0264-410X(01)00120-7] 3. Andersson MI, Maponga TG, Ijaz S, Theron G, Preiser W, Tedder RS. High HBV viral loads in HIV-infected pregnant women at a tertiary hospital, South Africa. J Acquir Immune Defic Syndr 2012;60(4):e111-el12. [http://dx.doi.org/10.1097/QAI.0b013e31825aeee7]

Department of Health, South Africa. The South African Antiretroviral Treatment Guidelines 2013. Date of implementation 1 April 2013. http://www.doh.gov.za/docs/policy/2013/ART_Treatment Guidelines_Final_25March2013.pdf (accessed 21 April 2013).

5. Spearman CWN, Sonderup MW, Botha JF, et al. South African guideline for the management of chronic hepatitis B: 2013. S Afr Med J 2013;103(5):337-349. [http://dx.doi.org/10.7196/SAMJ.6452]

6. Burnett RJ, Ngobeni JM, Francois G, et al. Increased exposure to hepatitis B virus infection in HIV-positive South African antenatal women. Int J STD AIDS 2007;18(3):152-156. [http://dx.doi. org/10.1258/095646207780132523

7. Chetty V, Moodey D, Chuturgoon A. Evaluation of a 4th generation rapid HIV test for earlier and reliable detection of HIV infection in pregnancy. J Clin Virol 2012;54(2):180-184. [http://dx.doi. org/10.1016/j.jcv.2012.02.021

8. Firnhaber C, Reyneke A, Schulze D, et al. The prevalence of hepatitis B co-infection in a South African (SA) urban government HIV clinic. S Afr Med J 2008;98(7):541-544.

9. Dusheiko GM, Conradie JD, Brink BA, Marimuthu T, Sher T. Differences in the regional prevalence of chronic hepatitis B in southern Africa - implications for vaccination. S Afr Med J 1989;75(10):473-478. 10. Kumar M, Singh T, Sinha S. Chronic hepatitis B virus infection and pregnancy. J Clin Exp Hepatol 2012;2(4):366-381. [http://dx.doi.org/10.1016/j.j.jeh.2012.09.001]

11. Cheung KW, Seto MTY, Wong SF. Towards complete eradication of hepatitis B infection from perinatal transmission: Review of the mechanisms of in utero infection and the use of antiviral treatment during pregnancy. European Journal of Obstetrics \& Gynecology and Reproductive Biology 2013;169(1):17-23. [http://dx.doi.org/10.1016/j.ejogrb.2013.02.001]

12. Department of Health, South Africa. Clinical Guidelines: PMTCT (Prevention of Mother to Child Transmission). 2010. http://www.fidssa.co.za/images/PMTCT_Guidelines.pdf (accessed 21 February 2013).

3. Dusheiko G. Interruption of mother-to-infant transmission of hepatitis B: Time to include selective antivira prophylaxis? Lancet 2012;379(9830):2019-2021. [http://dx.doi.org/10.1016/S0140-6736(11)61182-3]

4. Thorne C, Newell ML. HIV, hepatitis and pregnancy. Women's Health Medicine 2005;2(2):40-43. [http://dx.doi.org/10.1383/wohm.2.2.40.63064]

15. Mayaphi S, Rossouw TM, Masemola DP, Olorunju SAS, Mphahlele MJ, Martin DJ. HBV/ HIV co-infection: The dynamics of HBV in South African patients with AIDS. S Afr Med 2012;102(3):157-162.

16. Levy M, Koren G. Hepatitis B vaccine in pregnancy: Maternal and fetal safety. Am J Perinatol 1991;8(3):227-232. [http://dx.doi.org/10.1055/s-2007-999384]

17. Ayuk J, Mphahlele JM, Bessong P. Hepatitis B virus in HIV-infected patients in north-eastern South Africa: Prevalence, exposure, protection and response to HAART. S Afr Med J 2013;103(5):330-333. [http://dx.doi.org/10.7196/SAMJ.6304]

18. Hoffmann CJ, Thio CL. Clinical implications of HIV and hepatitis B co-infection in Asia and Africa. Lancet Infect Dis 2007;7(6):402-409. [http://dx.doi.org/10.1016/S1473-3099(07)70135-4] 